\title{
Astronomy from 80 Degrees North on Ellesmere Island, Canada
}

\author{
Eric Steinbring \\ National Research Council of Canada, \\ 5071 West Saanich Rd, Victoria, BC V9E 2E7, Canada \\ email: Eric.Steinbring@nrc-cnrc.gc.ca
}

\begin{abstract}
Site testing carried out on Ellesmere Island over recent years has shown that mountainous coastal terrain there can provide high clear-sky fractions in the long dark season, with low precipitable water-vapour column and prospects for excellent seeing. This presents new possibilities for time-domain and survey-mode science in the northern hemisphere, allowing uninterrupted high-precision photometry in the optical/near-infrared, but also gains in the submillimetre/millimetre. Efforts underway at the Eureka research station, at 80 degrees latitude, are reviewed. This location provides year-round access to a nearby site being developed as a pathfinder observatory. A program of variable-star and transient searches involving a wide-field imaging system has begun, with some early results. Plans include extrasolar-planet hunting via transit surveys, and future directions are discussed.
\end{abstract}

Keywords. arctic, site testing, telescopes, optical, submillimeter

\section{Introduction}

Ellesmere Island, in the territory of Nunavut, Canada, stretches beyond $82^{\circ} \mathrm{N}$ latitude at its far northern tip, near the military base of Alert. Some mountains here are the highest within 10 degrees of the Pole, topping 2,600 m at Barbeau Peak. Many coastal summits reach from $1,000 \mathrm{~m}$ to $1,900 \mathrm{~m}$, above a strong atmospheric thermal inversion that persists through the winter. Site testing on three of the four highest peaks within $100 \mathrm{~km}$ of the northwestern coast began in 2006. These locations are indicated by red crosses on the map in Figure 1. They are accessible by helicopter from the Eureka research base in summer, which allowed the installation of small autonomous weather stations and cameras for remote monitoring through winter (Steinbring et al. 2008; Wallace et al. 2008). Although the initial stations were power-limited - relying on wind-turbines and a fuel-cell - they were able to confirm the excellent weather conditions expected at these sites based on meteorology and satellite analysis: stable temperature near $-30^{\circ} \mathrm{C}$, clear-sky fractions approaching $70 \%$ and long periods of calm winds (Steinbring et al. 2010). And importantly, this established the feasibility of access and potential for developing and maintaining more advanced astronomical instrumentation at the highestelevation/highest-latitude mountains attainable (Steinbring et al. 2012b).

Comparison can also be made to a lower-elevation coastal location nearer to Eureka, the site of the Polar Environment Atmospheric Research Laboratory (PEARL; $80^{\circ} \mathrm{N}$, $600 \mathrm{~m}$ ). This manned facility, although less ideal than the remote sites, allows realistic demonstration of a variety of scientific programs at a High Arctic astronomical observatory. This review article summarizes the current status of that work near Eureka. Section 2 provides an outline of logistics and the currently-known astronomical sky properties, which is followed in Section 3 by a discussion of scientific programs being undertaken and possible future directions. 


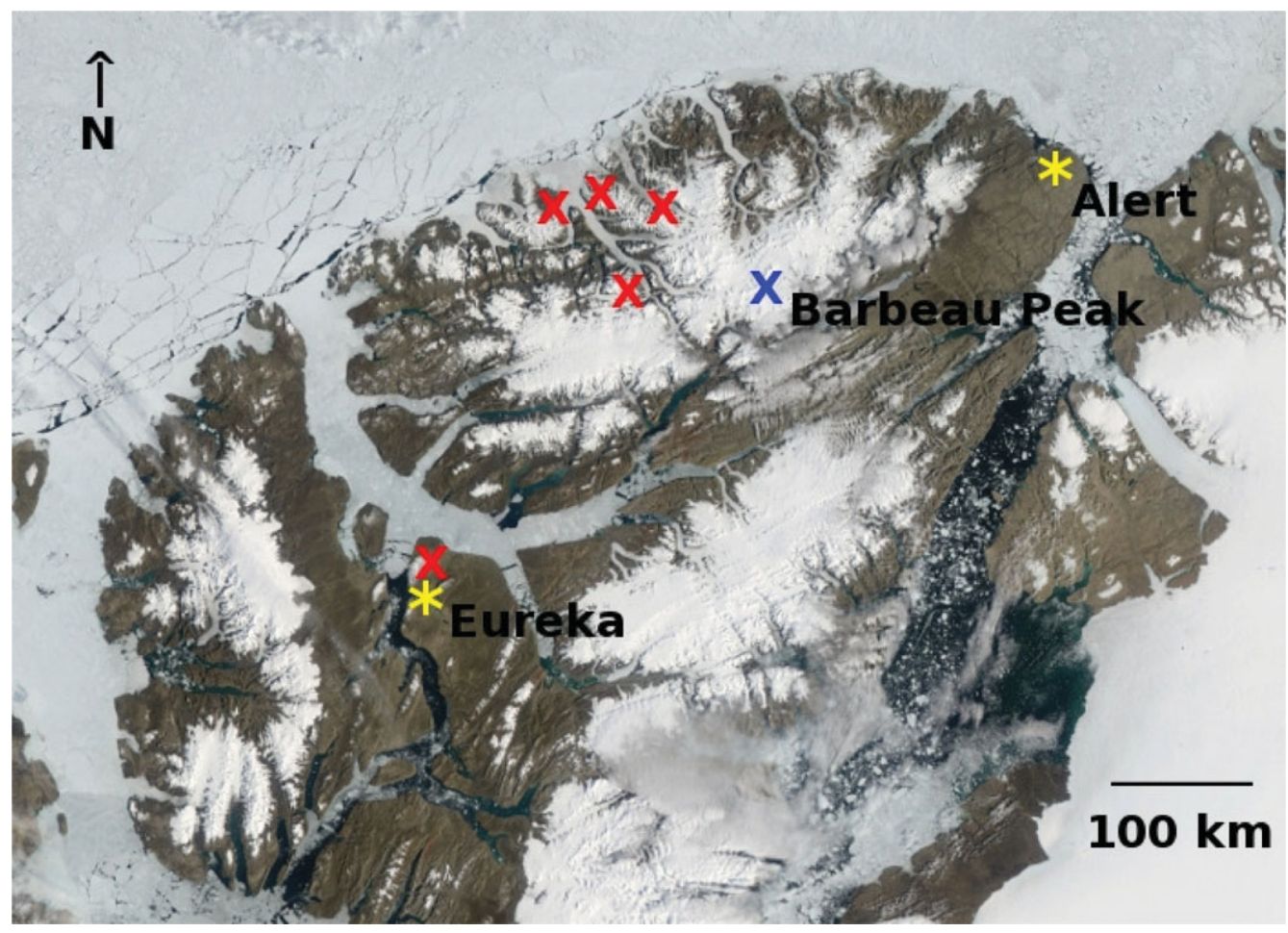

Figure 1. A map of northern Ellesmere Island; coastal mountains reach above $1,000 \mathrm{~m}$, the tallest, Barbeau Peak is over 2,600 m. Study sites are marked with red crosses; PEARL is nearest to Eureka. (Colour image available in the online version.)

\section{Site Testing and Development Near Eureka}

Eureka is a base of Environment Canada, the weather service of the Canadian federal government. The PEARL facility, to the northwest of Eureka at the end of a $15 \mathrm{~km}$ road, is shown in Figure 2. Although it was originally designed for atmospheric studies, particularly the monitoring of ozone using Laser Detection and Ranging (LIDAR), it also offers a good platform for small astronomical instruments. Power is supplied from diesel generators in Eureka, and the building provides a large, flat roof. Below this is warm laboratory space, dormitory areas and a kitchen. Broadband internet and technical support have been provided through the Canadian Network for the Detection of Atmospheric Change (CANDAC).

\subsection{Logistics}

The key advantages of Eureka and PEARL are existing infrastructure and the relative ease of access to high-elevation terrain nearby the ice-locked coast. Eureka has a 5,000 foot all-season runway which provides year-round access via commercial charters: small turboprop aircraft, but also including large transports, e.g. Boeing 737. In late summer, when ice conditions allow, a yearly re-supply vessel provides fuel and large cargo for the base facility. The weather station maintains a rotating staff of typically eight people: a manager, three weather technicians, a cook, a mechanic, a heavy-equipment operator, and a handyperson, although in summer the number of additional visitors can swell to 20 or 30 . PEARL can be accessed from here by truck in all seasons, and observers either go up to "the summit" periodically or stay for more extended periods. 

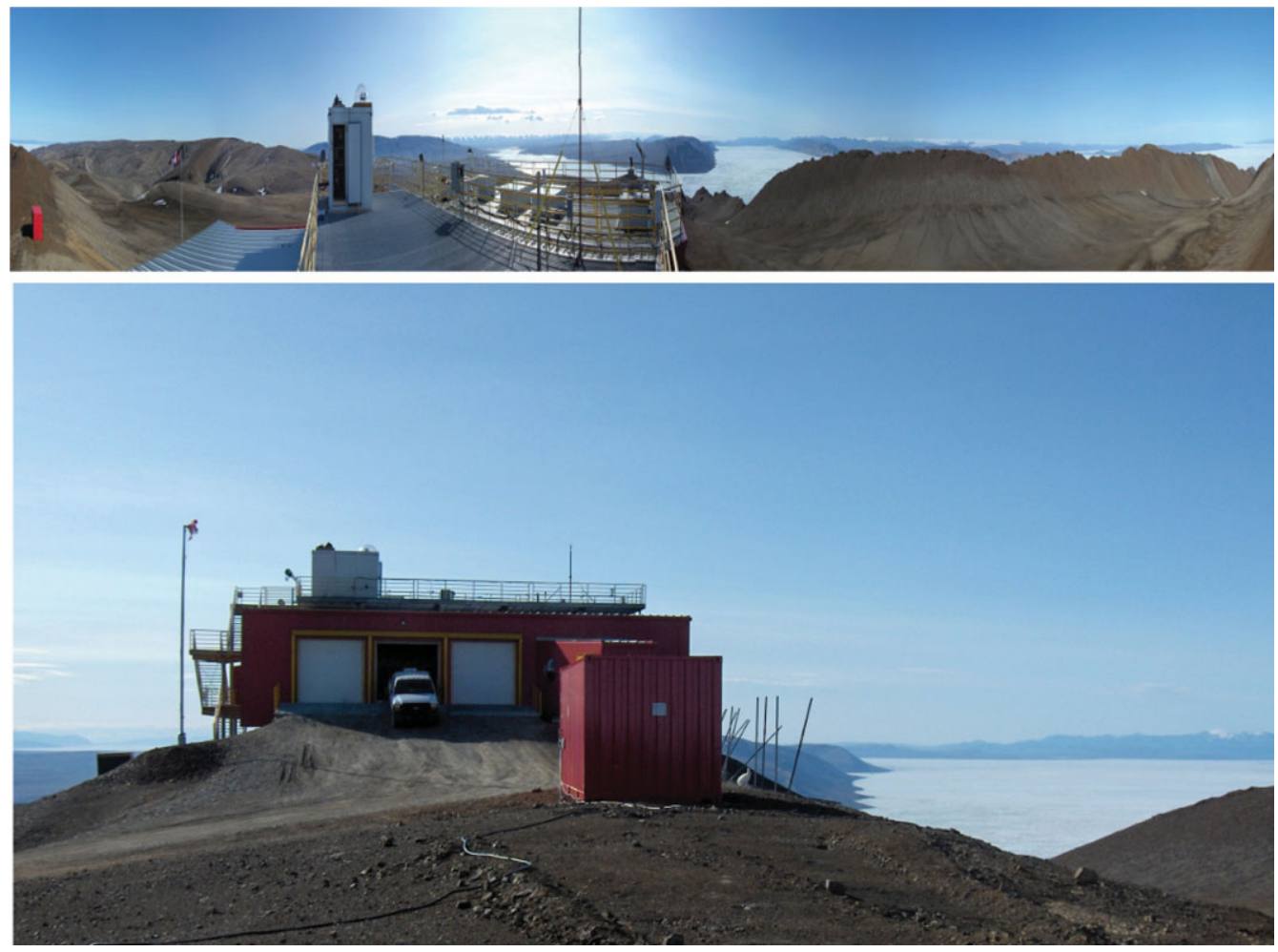

Figure 2. The PEARL facilty near Eureka; the observing platform is accessed with the external stairway at left. A 360 degree panoramic view (above) is from the observing platform itself. The orientation of both images is to the south, aligned with the mast of the rooftop meteorological station. Photos: Thomas Pfrommer.

\subsection{Clear-Sky Fraction}

Each hour at the Eureka weather station, a visual estimate of cloud cover is obtained by a technician as part of the standard meteorological record. There are over 50 years of continuous data which show that much of the time in winter is clear or with thin, uniform attenuation due to suspended ice crystals. More recently, a detailed study of cloud cover has been carried out using an all-sky-viewing camera installed on the PEARL roof, discussed in Steinbring et al. (2012a).

The PEARL All-Sky Imager (PASI) was designed for atmospheric studies, particularly buoyancy wave phenomenon, not astronomy. However, the central wavelength of one of its narrow-band filters is similar to V. Near-continuous photometry of Polaris over two winters provides a good estimate of sky clarity, after subtraction of variation in this wellknown Cepheid. About $84 \%$ of the time clouds, primarily in the form of ice crystals, have an attenuation less than $2 \mathrm{mag}$, up to $68 \%$ of the time is clear (below $0.5 \mathrm{mag}$ ) which can persist for over 100 hours at a time; and $48 \%$ of that time is truly photometric (no cloud at all).

Interestingly, the PASI data are also consistent with the expectations from LIDAR and RADAR observations that the density of ice crystals decreases with elevation, under calm conditions. If correct, some higher terrain near Eureka should have clearer conditions than those experienced at PEARL (Steinbring et al. 2012a). 


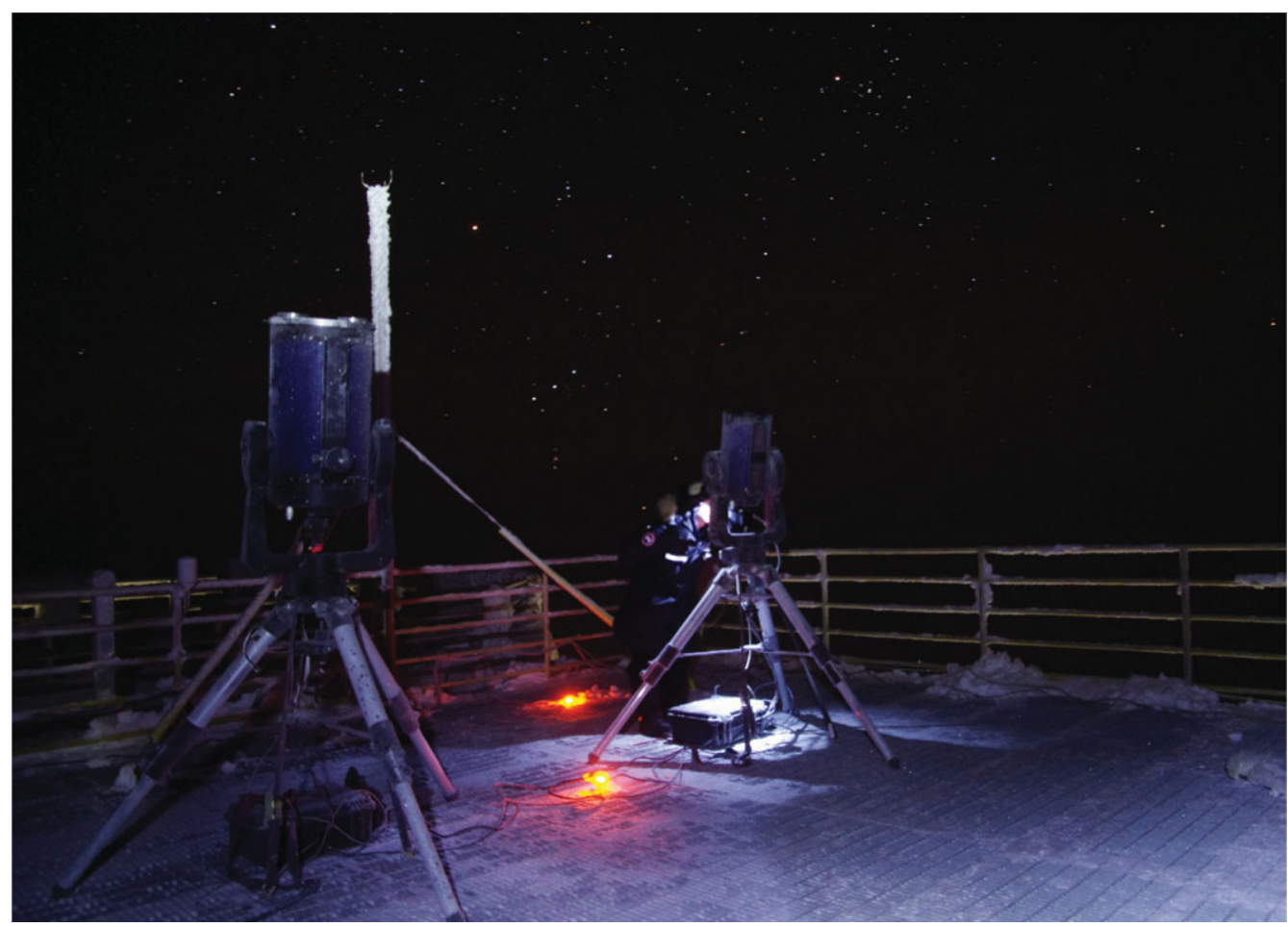

Figure 3. An observer checking on DIMM and MASS/DIMM site-testing telescopes deployed on the PEARL observing platform. Behind is the ice-covered mast of the meteorological station, beyond and to the right is the constellation Orion. Photo: Pierre Fogal.

\subsection{Sub-Millimetre Opacity}

The precipitable water vapour column at sea level in winter for Eureka is very low, with a median under $2 \mathrm{~mm}$, so it should not be a surprise that millimetre-wave opacity is good. This was verified using a $225 \mathrm{GHz}$ tipping radiometer, deployed on the PEARL observing platform for three months in the winter/spring of 2011. The main result was that the opacity was $\tau=0.1$, and stable with temperature and humidity (Asada et al. 2012). This is also discussed in more detail by Matsushita et al. (2012) in this Proceedings.

\subsection{Sky Brightness and Aurorae}

Sky brightness has been measured from photometry with PASI and using a commercial single-diode device. These agree that without a moon, sky brightness reaches a minimum for Sun elevations just below -12 degrees of 21 mag per square-arcsec, as expected for the latitude of Eureka (Steinbring et al. 2012a; Sivanandam et al. 2012). Eureka is within the auroral hole; when aurorae are seen, they are typically along the southern horizon. There is no evidence of strong aurorae in the PASI data, although that would not be anticipated for the filter bandpass. Initial measurements at J-band also suggest nearinfrared skies are as dark as the best mid-latitude sites (Sivanandam et al. 2012), with further observations planned in upcoming winters.

\subsection{Seeing}

Ground-layer turbulence measurements sensitive up to $100 \mathrm{~m}$ have been made from the PEARL rooftop using an autonomous lunar scintillometer: the Arctic Turbulence Profiler (ATP). These show a thin boundary layer, with a median contribution to total seeing 


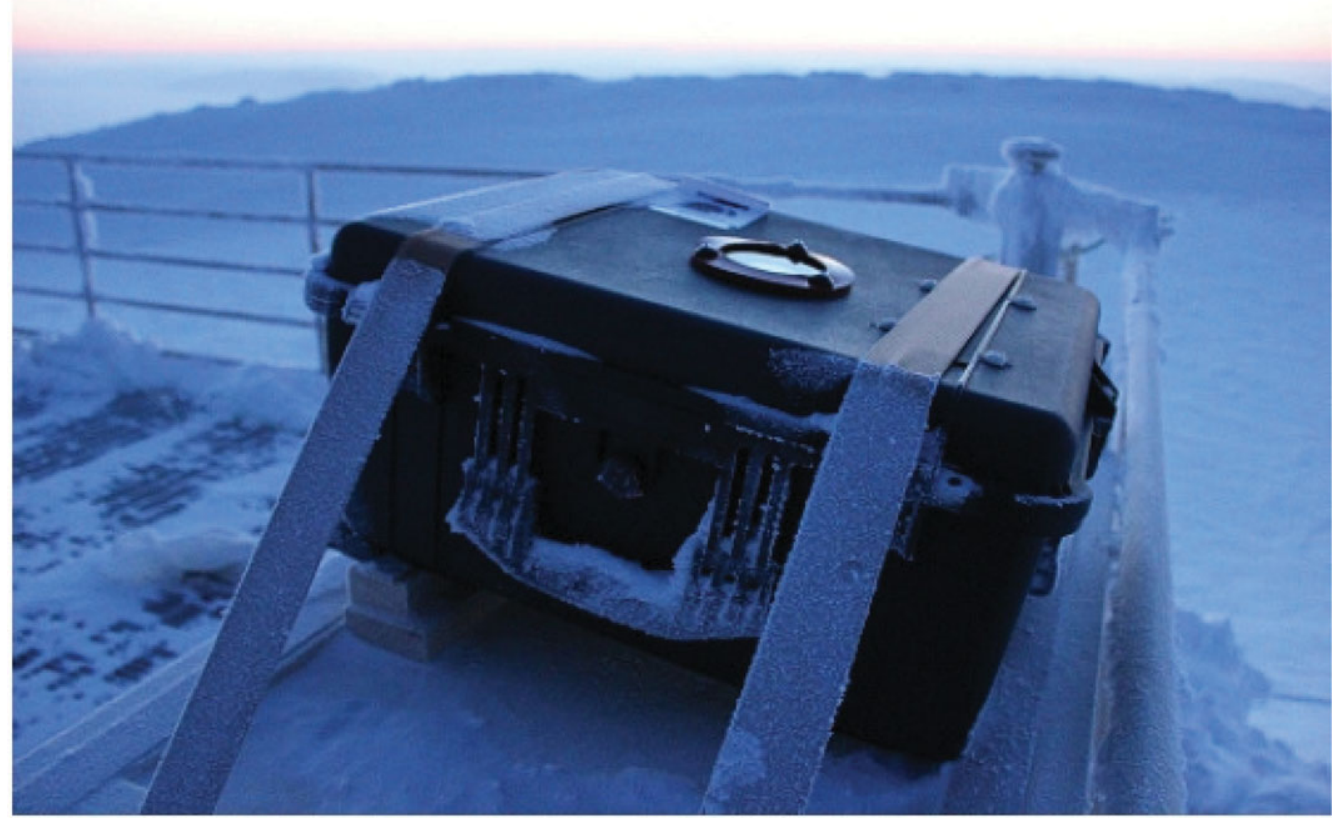

Figure 4. An AWCam deployed on the PEARL observatory platform. Photo: Wayne Ngan.

of 0.5 arcsec at an elevation of $15 \mathrm{~m}$ (Hickson et al. 2010), comparable to the best midlatitude sites.

Because northern Ellesmere is usually within the Arctic polar vortex, with the jet stream well to the south, the expectation is that Eureka will also benefit from excellent free-seeing - analogous to atmospheric conditions already demonstrated on the Antarctic glacial plateau (Lawrence et al. 2004). To confirm this, Differential Image Motion Monitor (DIMM) and Multi-Aperture Scintillation Sensor (MASS) observations have been obtained during two campaigns in winter 2011 and 2012 from the PEARL roof. Seeing of 0.6 arcsec to 0.9 arcsec is typical, consistent with the ATP results plus weak free-atmosphere seeing, although there is still not sufficient data for solid statistics. Obtaining data over a longer period, using an autonomous system on a standard 6-m tall tower away from the building should be conclusive, and is planned.

\section{Initial Science and Future Directions}

Astronomical studies requiring steady, uninterrupted observations are well suited to a near-PEARL site. The cold and dry conditions lead to long periods of low opacity in winter darkness, and at these latitudes high-elevation targets maintain essentially constant airmass. By taking advantage of the ease of accessibility afforded by the location, efforts can focus on open-shutter efficiency. Taken together, this can be a particularly useful path towards wide-field surveys and time-domain scientific programs, such as highcadence surveillance of variable or transient objects in the optical. But other science can also benefit, from the UV through to the millimetre.

So far, the main scientific pursuit being considered near PEARL is the detection of extra-solar planets via transits. One way to exploit the persistence of relatively uniform and thin sky transparency is to monitor large areas of sky. A first test of this approach 
was obtained with the Arctic Wide-field Cameras (AWCams) deployed in winter 2012, observing a fixed field centred on the celestial North Pole. One of these instruments is shown in Figure 4. The details of the design can be found in Law et al. (2012) and first results will appear in the literature soon: millimag-level photometry of many bright stars near Polaris, including known eclipsing binary W UMi. A logical extension of the AWCams called the Compound Arctic Telescope Survey (CATS) is to expand the field by deploying multiple unit cameras, with a rotating mount counteracting star trails and allowing coverage of the entire sky down to airmass of about 2. In complement, a larger aperture telescope monitoring a smaller field may realize the benefits of good seeing. The 0.5-m aperture Dunlap Arctic Telescope (DIAT), also discussed in Law et al. (2012), is planned for deployment on the ground nearby PEARL. Again, high-cadence millimag photometry would be the focus of a survey of $\mathrm{M}$ dwarf stars, with an advantage of the long twilight at this latitude possibly allowing multiple transit detections during a single winter season.

A larger, future optical/near-infrared telescope might take full advantage of the thin boundary layer and expected weak free-atmospheric seeing. A concept similar to that proposed by Rene Racine over 20 years ago for the site now occupied by Gemini North was outlined in a White Paper by Carlberg, Hickson, \& Steinbring (2010), employing low-order adaptive optics to achieve wide-field diffraction-limited performance. This $1 \mathrm{~m}$ to $4 \mathrm{~m}$-class facility is endorsed in the 2010 Canadian Long Range Plan for Astronomy (www.casca.ca/lrp2010/), which recommends a design study once site testing is completed.

\section{References}

Asada, K., Martin-Cocher, P. L., Chen C.-P., Matsushita, S., Chen, M.-T., Inoue, M., Huang, Y.-D., Inoue, M., Ho, P. T. P., Paine, S. N., \& Steinbring, E., 2012, SPIE Conf. Series, in press

Carlberg, R., Hickson, P., \& Steinbring, E., 2010, White Paper Super Seeing Sites in the Canadian High Arctic

Hickson, P., Carlberg, R., Gagne, R., Pfrommer, T., Racine, R., Schoeck, M., Steinbring, E., \& Travouillon, T., 2010, SPIE Conf. Series, 7733, 53

Law, N., Sivanandam, S., Murowinski, R., Carlberg, R., Ahmadi, P., Steinbring, E., \& Graham, J., 2012, SPIE Conf. Series, in press

Lawrence, J. S. et al. 2004, Nature, 431, 238

Matsushita, S., Chen, M.-T., Martin-Cocher, P., Asada, K., Chen, C.-P., Inoue, M., Paine, S., Turner, D., \& Steinbring, E., 2012, These Proceedings

Sivanandam, S., Tekatch, A., Welch, D., Abraham, B., Graham, J., \& Steinbring, E., 2012, SPIE Conf. Series, in press

Steinbring, E., Leckie, B., Welle, P., Hardy, T., Cole, B., Bayne, D., Croll, B., Walker, D. E., Carlberg, R. G., Fahlman, G. G., Wallace, B., \& Hickson, P., 2008, SPIE, 7012, 1

Steinbring, E., Carlberg, R., Croll, B., Fahlman, G., Hickson, P., Leckie, B., Pfrommer, T., \& Schoeck, M., 2010, PASP, 122, 1092

Steinbring, E., Ward, W., \& Drummond, J. R., 2012, PASP, 124, 185

Steinbring, E., Leckie, B., Hardy, T., Caputa, K., \& Fletcher, M., 2012, SPIE Conf. Series, in press

Wallace, B., Steinbring, E., Fahlman, G., Leckie, B., Hardy, T., Fletcher, M., Pennington, M., Caputa, K., Carlberg, R., Croll, B., Bayne, D., Cole, B., Hickson, P., Pfrommer, T., \& Thorsteinson, S., 2008, AMOS Conf. Series, Ed. S. Ryan, 7, The Maui Economic Development Board 